University of South Carolina

Scholar Commons

$1-17-2000$

\title{
Selective Area Deposited Blue GaN-InGaN Multiple-Quantum Well Light Emitting Diodes over Silicon Substrates
}

\author{
J. W. Yang
}

A. Lunev

Grigory Simin

University of South Carolina - Columbia, simin@engr.sc.edu
A. Chitnis
M. Shatalov

See next page for additional authors

Follow this and additional works at: https://scholarcommons.sc.edu/elct_facpub

Part of the Electromagnetics and Photonics Commons

\section{Publication Info}

Published in Applied Physics Letters, Volume 76, Issue 3, 2000, pages 273-275.

(C)Applied Physics Letters 2000, American Institute of Physics (AIP).

Yang, J. W., Lunev, A., Simin, G., Chitnis, A., Shatalov, M., Khan, M. A., Van Nostrand, J. E., \& Gaska, R. (17 January 2000). Selective Area Deposited Blue GaN-InGaN Multiple-Quantum Well Light Emitting Diodes over Silicon Substrates. Applied Physics Letters, 76 (3), 273-275. http://dx.doi.org/10.1063/1.125745

This Article is brought to you by the Electrical Engineering, Department of at Scholar Commons. It has been accepted for inclusion in Faculty Publications by an authorized administrator of Scholar Commons. For more information, please contact digres@mailbox.sc.edu. 


\section{Author(s)}

J. W. Yang, A. Lunev, Grigory Simin, A. Chitnis, M. Shatalov, M. Asif Khan, Joseph E. Van Nostrand, and R. Gaska 


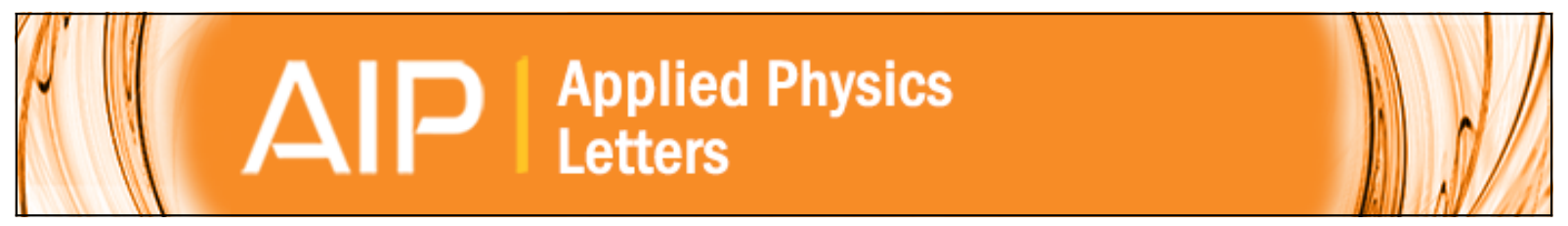

Selective area deposited blue GaN-InGaN multiple-quantum well light emitting diodes over silicon substrates

J. W. Yang, A. Lunev, G. Simin, A. Chitnis, M. Shatalov, M. Asif Khan, Joseph E. Van Nostrand, and R. Gaska

Citation: Applied Physics Letters 76, 273 (2000); doi: 10.1063/1.125745

View online: http://dx.doi.org/10.1063/1.125745

View Table of Contents: http://scitation.aip.org/content/aip/journal/apl/76/3?ver=pdfcov

Published by the AIP Publishing

\section{Articles you may be interested in}

The effect of silicon doping in the barrier on the electroluminescence of InGaN/GaN multiple quantum well light emitting diodes

J. Appl. Phys. 114, 103102 (2013); 10.1063/1.4820450

Study of the dominant luminescence mechanism in In Ga N/Ga N multiple quantum wells comprised of ultrasmall InGaN quasiquantum dots

Appl. Phys. Lett. 86, 121915 (2005); 10.1063/1.1891291

Comparison of blue and green In Ga N/Ga N multiple-quantum-well light-emitting diodes grown by metalorganic vapor phase epitaxy

Appl. Phys. Lett. 86, 101903 (2005); 10.1063/1.1866634

InGaN multiple-quantum-well green light-emitting diodes on Si grown by metalorganic chemical vapor deposition J. Appl. Phys. 91, 528 (2002); 10.1063/1.1408264

GalnN/GaN multiple-quantum-well light-emitting diodes grown by molecular beam epitaxy

Appl. Phys. Lett. 74, 3616 (1999); 10.1063/1.123199

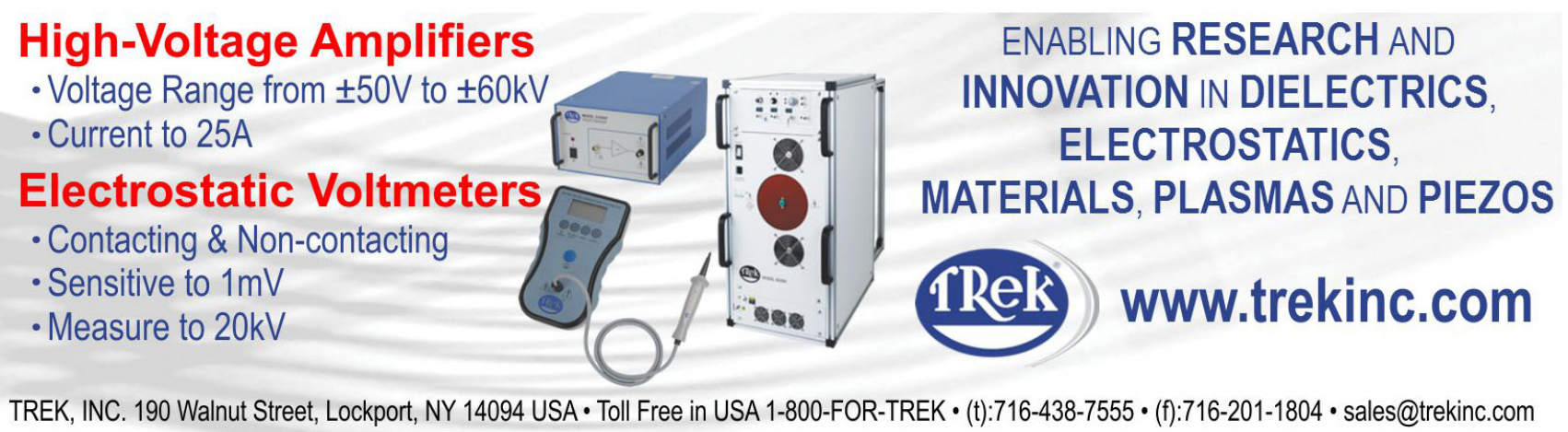




\title{
Selective area deposited blue GaN-InGaN multiple-quantum well light emitting diodes over silicon substrates
}

\author{
J. W. Yang, A. Lunev, G. Simin, A. Chitnis, M. Shatalov, and M. Asif Khan ${ }^{\text {a) }}$ \\ Department of Electrical and Computer Engineering, University of South Carolina, Columbia, \\ South Carolina 29028 \\ Joseph E. Van Nostrand \\ Materials and Manufacturing Directorate, AFRL/MLPA Bldg. 620, 2241 Avionics Circle, Ste. 21, \\ Wright-Patterson AFB, Ohio 45433 \\ R. Gaska \\ Sensor Electronic Technology, Inc., Troy, New York 12180
}

(Received 28 September 1999; accepted for publication 17 November 1999)

\begin{abstract}
We report on fabrication and characterization of blue $\mathrm{GaN}-\mathrm{InGaN}$ multi-quantum well (MQW) light-emitting diodes (LEDs) over (111) silicon substrates. Device epilayers were fabricated using unique combination of molecular beam epitaxy and low-pressure metalorganic chemical vapor deposition growth procedure in selective areas defined by openings in a $\mathrm{SiO}_{2}$ mask over the substrates. This selective area deposition procedure in principle can produce multicolor devices using a very simple fabrication procedure. The LEDs had a peak emission wavelength of $465 \mathrm{~nm}$ with a full width at half maximum of $40 \mathrm{~nm}$. We also present the spectral emission data with the diodes operating up to $250^{\circ} \mathrm{C}$. The peak emission wavelengths are measured as a function of both dc and pulse bias current and plate temperature to estimate the thermal impedance. (C) 2000 American Institute of Physics. [S0003-6951(00)01103-7]
\end{abstract}

Due to their low cost and electronics integration potential, silicon substrates are a challenging choice for fabrication of light-emitting diodes (LED) and laser devices based on direct band gap III-V materials. However, in spite of significant research effort, the progress toward fabricating medium band gap III-V devices over silicon substrates has been limited. The key stumbling block has been the lattice mismatch related defects, which significantly reduce the lifetimes of light emission devices. In contrast, $\mathrm{GaN}-\mathrm{InGaN}$ based LEDs grown on sapphire have been shown to have lifetimes in excess of $10^{4} \mathrm{~h}^{1}{ }^{1}$ in spite of around $10^{8}-10^{10}$ threading dislocations per $\mathrm{cm}^{2}$. Indeed, blue-green $\mathrm{GaN}-\mathrm{InGaN}$ multiquantum wells (MQW) LEDs over sapphire and silicon carbide substrates are now a successful commercial product. It can thus be postulated that performance of $\mathrm{GaN}$ based light emission devices on silicon may not be significantly affected by the presence of lattice mismatch related defects. In the past, Guha $e t$ al. $^{2}$ have reported on an impurity-band purple $\mathrm{GaN}-\mathrm{AlGaN}$ double heterostructure (DH) LED on (111) Si substrate. They used molecular beam epitaxy (MBE) for the growth process. Using the relatively low temperature MBE process for the LED layers avoids the outdiffusion and auto doping from Si substrate. However, low temperature growths also degrade the luminous efficiency of III-V based quantum wells and superlattices. While this manuscript was under preparation Tran et al. also reported on a blue $\mathrm{InGaN} / \mathrm{GaN}$ MQW LED over Si (Ref. 3) using metalorganic chemical vapor deposition (MOCVD) process. We now report on using a unique combination of MBE and low-pressure metalorganic chemical vapor deposition (LPMOCVD), to fabri-

a)Electronic mail: asif@engr.sc.edu cate a blue GaN-InGaN MQW LED over (111) Si, with a peak emission wavelength at $465 \mathrm{~nm}$. We also deposited the device epilayers only in selective areas defined by openings in a $\mathrm{SiO}_{2}$ mask. Our MBE/MOCVD combination procedure avoids silicon outdiffusion and autodoping because of the relatively low growth temperature used for the AlN buffer layer $\left(800^{\circ} \mathrm{C}\right)$. Also, in contrast to MOCVD, the MBE technique avoids the formation of silicon nitride layer on the silicon surface. MBE grown AlN buffer is a high quality single crystal layer. Furthermore our use of selective area MOCVD for the fabrication of the LED epilayers not only makes processing simple but also opens up the possibility of sequential growth for LEDs with multicolor pixels and heterointegration of GaN-based optoelectronic devices with $\mathrm{Si}$ based electronics. We also demonstrate the operation of these selective area deposited LEDs (over Si substrates) at temperatures in excess of $250^{\circ} \mathrm{C}$ and use our data to estimate the device thermal impedance.

The epilayer structure for our GaN-InGaN MQW LED consists of a $100 \AA$ thick AlN buffer layer that is deposited over the (111) $n^{+} \mathrm{Si}$ substrates using $\mathrm{NH}_{3}$ in a gas-source MBE system. The MBE growth is carried out at $800{ }^{\circ} \mathrm{C}$ using an $\mathrm{Al}$ flux to have a growth rate of $0.3 \mathrm{ML} \mathrm{s}^{-1}$. Prior to growth of the AlN layer, one monolayer of $\mathrm{Al}$ is deposited followed by exposure to $\mathrm{NH}_{3}$ for several seconds. This was found to reduce the formation of amorphous $\mathrm{SiN}$ which degrades the quality of the AlN buffer layer and subsequently the nitride device. The AlN layers from the above process exhibited a $1 \times 1$ RHEED pattern with sharp streaks and weak Kikuchi lines.

Following the AlN buffer layer a $0.2 \mu \mathrm{m}$ thick Si doped $n^{+}$GaN layer $\left(n^{+} \sim 1 \times 10^{18} \mathrm{~cm}^{-3}\right)$ was deposited using low pressure MOCVD. Triethylgallium $(8.5 \mu \mathrm{moles} / \mathrm{min})$ and 


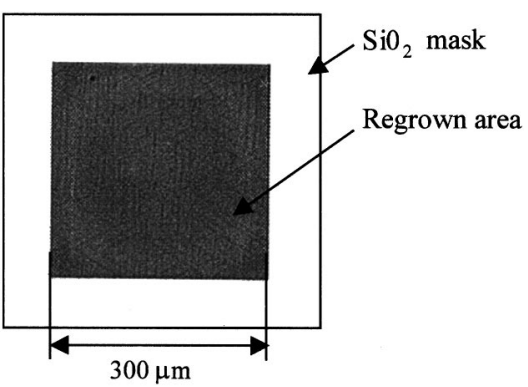

FIG. 1. The surface image for Si-based $300 \times 300 \mu \mathrm{m}^{2}$ selective area grown InGaN-GaN MQW structure after removing the $\mathrm{SiO}_{2}$ mask.

$\mathrm{NH}_{3}$ (1 slm) were used as the precursors and the growth temperature and pressure were respectively $900{ }^{\circ} \mathrm{C}$ and 76 Torr. We then measured the electrical conductivity across the $n^{+}$-Si substrate and the (AlN/GaN) heterojunction along the (111) direction. For this measurement $300 \AA$ diameter Ti (50 $\AA) / \mathrm{Al}(100 \AA) / \mathrm{Ti}(200 \AA) / \mathrm{Au}(1000 \AA)$ contacts were deposited on the $n^{+}$GaN layer. The entire backside of the $n^{+}-\mathrm{Si}$ substrate was also metallized using the same metals scheme. These $n$-type ohmic contacts were annealed at $700^{\circ} \mathrm{C}$ for 1 min in a $\mathrm{N}_{2}$-ambient using a rapid thermal annealer (RTA). The $I-V$ measurements show a $0.5 \mathrm{~V}$ barrier with a differential resistance of $50 \Omega$ after turn-on. This barrier can be due to the band offsets between the (111) Si and the AlN buffer layer heterojunction. Similar to Guha et al. ${ }^{2}$ we also believe the growth of $n^{+} \mathrm{GaN}$ in the voids in our AlN buffer layer to provide the pathway for the vertical conduction.

Subsequently, we deposited a $0.2 \mu \mathrm{m}$ thick $\mathrm{SiO}_{2}$ layer on the $\mathrm{Si} /(\mathrm{AlN} / \mathrm{GaN})$ structures and opened up $300 \mu \mathrm{m}$ square via holes using a $\mathrm{HF} / \mathrm{H}_{2} \mathrm{O}_{2}$ solution and standard photolithography procedures. The samples were then reloaded in the LPMOCVD system and a $500 \AA$ thick $n$-GaN layer $(n$ $\left.=5 \times 10^{17} \mathrm{~cm}^{-3}\right)$, a GaN-InGaN MQW, and a $0.15 \mu \mathrm{m}$ thick $p$-GaN $\left(p=5 \times 10^{17} \mathrm{~cm}^{-3}\right)$ cap layer were selectively deposited in the $\mathrm{SiO}_{2}$ mask openings. The MQW region consisted of four $30 \AA$ thick $\mathrm{In}_{0.22} \mathrm{Ga}_{0.78} \mathrm{~N}$ quantum wells surrounded by $30 \AA$ thick $\mathrm{GaN}$ barrier layers. Under our growth conditions we did not observe any nitride material deposition over the $\mathrm{SiO}_{2}$ mask region. In Fig. 1 we include the surface image for $300 \times 300 \mu \mathrm{m}$ regrown area of InGaN-GaN MQW structure after removing $\mathrm{SiO}_{2}$ mask. The density of cracks, the surface roughness, and morphology of the regrown material are very close to those typically obtained in flat grown InGaN/GaN layers on $\mathrm{SiC}$ substrates. The selective area grown MQW LED structures were then metallized to form $p$-ohmic contacts. The top $p$ contact consisted of $\mathrm{Pd}$ (50 $\AA) / \mathrm{Au}(100 \AA)$ and it was annealed at $450^{\circ} \mathrm{C}$ in oxygen ambient. We measured it to have a $70 \%$ transparency in the blue-green wavelength region. The top contact area is 280 $\times 280 \mu \mathrm{m}^{2}$. Stripe-shaped Ti $(200 \AA / \mathrm{Au}(2000 \AA)$ probe contacts were then formed on the top of semitransparent $p$ contact to create contact pads with low spreading resistance. The $n$ contact to the (111) $n^{+}$Si substrate was formed using $\mathrm{Ti}(50 \AA) / \mathrm{Al}(100 \AA) / \mathrm{Ti}(200 \AA) / \mathrm{Au}(1000 \AA)$ and a $750^{\circ} \mathrm{C}$, 1 min anneal in forming gas.

In Fig. 2 we present the spectral emission of the LED (from the top $p$-contact side). Data are included for room temperature operation at a bias current of $20 \mathrm{~mA}$. The peakemission wavelength $(465 \mathrm{~nm})$ agrees well with that of the

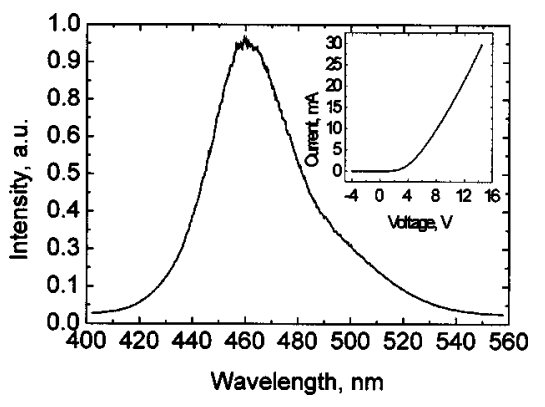

FIG. 2. The spectral emission of the InGaN/GaN LED on Si at room temperature for a bias current of $20 \mathrm{~mA}$. Inset: Room temperature $I-V$ curves for a $300 \mu \mathrm{m} \times 300 \mu \mathrm{m}$ MQW LED structure.

room temperature photoluminescence (PL) peak. For the PL measurements we used the MQW samples without the top $p$ layer and a cw-He-Cd laser. The LED full width at half maximum (FWHM) of $40 \mathrm{~nm}$ also compares favorably with that of the PL peak linewidth. The inset of Fig. 2 shows the current-voltage $(I-V)$ characteristic of the GaN-InGaN LED. A forward turn-on voltage of $3.2 \mathrm{~V}$ was measured. This value included the $0.5 \mathrm{~V}$ contribution from the $\mathrm{Si} /(\mathrm{AlN} /$ $\mathrm{GaN}$ ) heterojunction barrier and hence is comparable to the values typically reported for GaN-InGaN MQW LEDs over sapphire ${ }^{4}$ and $\mathrm{SiC}$ substrates. ${ }^{5}$ The forward differential resistance was measured to be around $250 \Omega$. This is approximately a factor of four higher than what we routinely obtain on our high-quality GaN-InGaN MQW LEDs over sapphire substrates. The low doping of the $p$-layer, $p$-contact resistivity, the differential resistance of AlN/Si interface, and possibly a counter doping of silicon from the $\mathrm{SiO}_{2}$ mask (used for the selective area deposition) may be responsible for this increase. Further work is needed to determine the exact cause for this increase.

In Fig. 3 we include the LED forward-bias $I-V$ characteristics from 25 to $250^{\circ} \mathrm{C}$ which establish that our device can sustain high temperature operation (up to $250^{\circ} \mathrm{C}$ ). We observe a decrease in the turn-on voltage and an increase in the forward-bias current with an increase of temperature. We also measured the spectral emission at temperatures from 25 to $250{ }^{\circ} \mathrm{C}$. We observed the peak emission wavelength to redshift with increasing temperature. The emission intensity

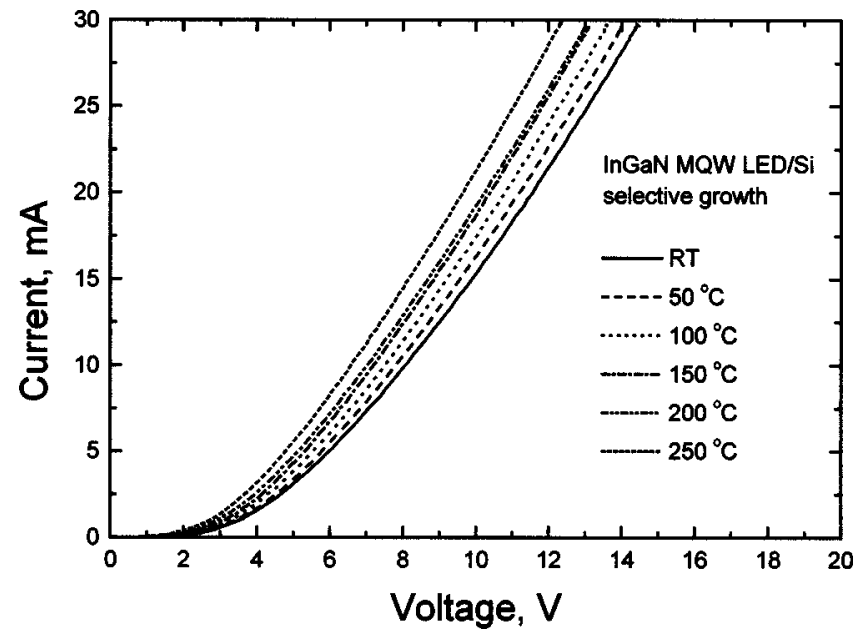

FIG. 3. Forward-bias $I-V$ characteristics from 25 to $250^{\circ} \mathrm{C}$ ambient temperature for InGaN/GaN MQW LED selectively grown on (111) $\mathrm{Si}$. 

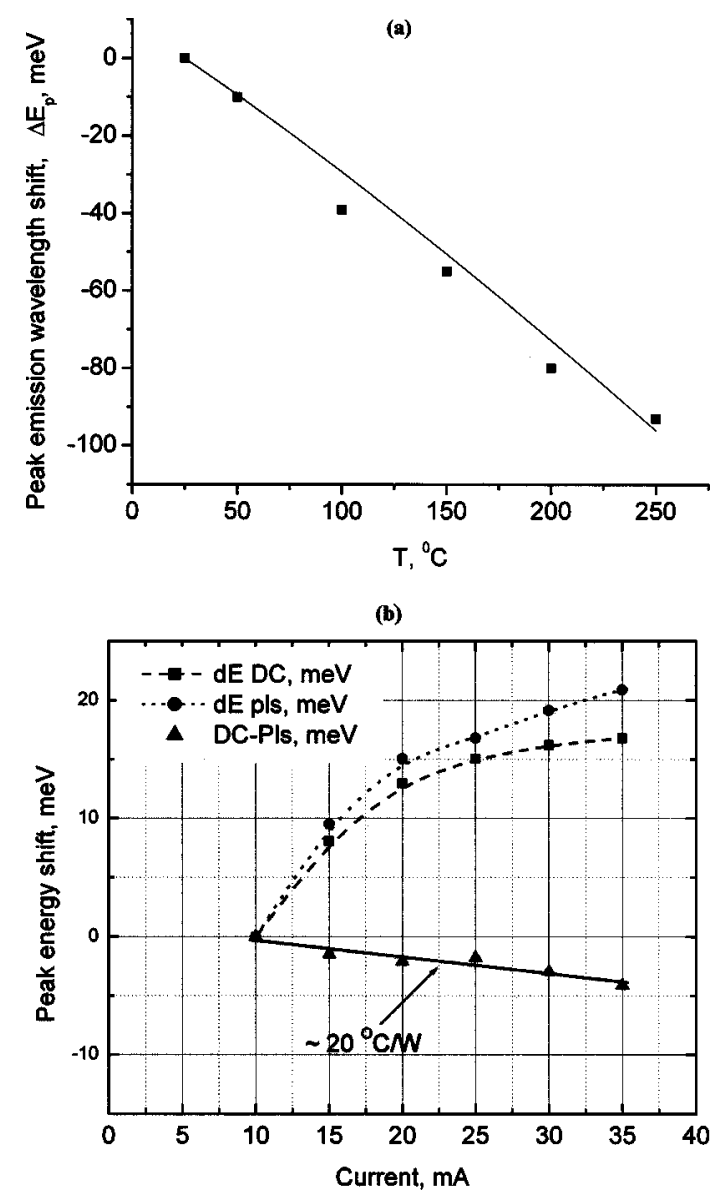

FIG. 4. (a) The peak emission energy shift as a function of temperature for the MQW LED on $\mathrm{Si}$, (b) the peak emission energy shift as a function of dc and pulsed pumping currents.

also decreased by a factor of 10 with an increase in temperature from 25 to $250^{\circ} \mathrm{C}$. Since the data of Fig. 3 show the temperature increase to result in increased forward bias current (for same voltage), this decrease in the emission intensity has to occur from degradation in the emission properties of the quantum well region. Included in Fig. 4(a) is the MQW LED peak emission energy shift as a function of temperature (solid circles). We have also plotted the estimated values of the emission energy shift due to band-gap narrowing from temperature increase (solid line). As seen, the redshift of our peak emission wavelength can thus be explained by this band-gap narrowing. The linewidth for the blue emission $(465 \mathrm{~nm})$ also increased from $40 \mathrm{~nm}$ at $25^{\circ} \mathrm{C}$ to $50 \mathrm{~nm}$ at $250^{\circ} \mathrm{C}$.

We then estimated the thermal impedance of our Sibased LED structures. For this we first measured the peak emission wavelength as a function of dc-pump current from 10 to $35 \mathrm{~mA}$. Increasing pump current density resulted in a blue shift in the peak emission wavelength. These data are indicated using square symbols (dashed curve) in Fig. 4(b). As previously reported ${ }^{6}$ we assign to band filling effect in the MQW region. To further isolate the contribution of device heating (which results in a redshift), we measured the peakwavelength shift using pulsed current pumping. The pulse width was $100 \mathrm{~ns}$. This resulted in increased blueshifts with an increase in the bias-current (again from 10 to $35 \mathrm{~mA}$ ). Circles (dotted line) in Fig. 4(b) show these data. We attribute the difference between the blueshifts under pulsed and dc pumping to the difference in device temperatures [see solid line with triangles in Fig. 4(b)]. The decrease of the blueshift at $35 \mathrm{~mA}$ bias current $(20 \mathrm{~V}$ forward bias) was estimated to be $4 \mathrm{meV}$. This from the data of Fig. 4(a), translates to a device temperature rise of $15^{\circ} \mathrm{C}$. From the input power and this temperature rise value we then estimated the thermal impedance of our device to be around $20^{\circ} \mathrm{C} / \mathrm{W}$. This agrees well with the estimated value of thermal impedance $R_{t}=L /(A k)=20^{\circ} \mathrm{C} / \mathrm{W}$. Here $L$ is the thickness of $\mathrm{Si}$ substrate $(300 \mu \mathrm{m}), A$ is the device area $(300 \mu \mathrm{m} \times 300 \mu \mathrm{m})$, and $k=1.5 \mathrm{~W} /(\mathrm{cm} \mathrm{K})$ is the thermal conductivity of silicon.

In summary we report on a blue $\mathrm{GaN}-\mathrm{In}_{0.22} \mathrm{Ga}_{0.78} \mathrm{~N}$ MQW LED with peak emission wavelength of $465 \mathrm{~nm}$ over (111)Si. A unique MBE/MOCVD combination process was used for the deposition of device epilayers over the Si substrate. We have also shown selective area epitaxy to be a viable process for depositing $\mathrm{GaN}-\mathrm{InGaN} \mathrm{MQW}$ based light-emitting devices with operation temperatures up to $250^{\circ} \mathrm{C}$. This process allows for the heterointegration of multicolor GaN-based LEDs with Si-based electronics.

This work was supported by the Ballistic Missile Defense Organization (BMDO) under Army SSDC Contract No. DASG60-97-C 0066, monitored by Dr. Brian Strickland and Dr. Kepi Wu.

${ }^{1}$ S. Nakamura, M. Senoh, S.-I. Nagahama, T. Matsushita, H. Kiyoku, Y. Sugimoto, T. Kozaki, H. Umemoto, M. Sano, and T. Mukai, Jpn. J. Appl. Phys., Part 2 38, L226 (1999).

${ }^{2}$ S. Guha and N. A. Bojarczuk, Appl. Phys. Lett. 72, 415 (1998).

${ }^{3}$ C. A. Tran, A. Osinski, R. F. Karlicek, Jr., and I. Berishev, Appl. Phys. Lett. 75, 1494 (1999).

${ }^{4}$ S. Nakamura, M. Senoh, N. Iwasa, and S.-I. Nagahama, Appl. Phys. Lett. 67, 1868 (1995).

${ }^{5}$ V. A. Dmitriev, K. G. Irvine, J. A. Edmond, C. H. Carter, Jr., N. I. Kuznetsov, A. S. Zubrilov, E. V. Kalinina, and D. V. Tsvetkov, Inst. Phys. Conf. Ser. 142, 1019 (1995).

${ }^{6}$ S. Nakamura, J. Vac. Sci. Technol. A 13, 705 (1995). 